PROCEEDINGS OF THE

AMERICAN MATHEMATICAL SOCIETY

Volume 137, Number 8, August 2009, Pages 2749-2754

S 0002-9939(09)09838-4

Article electronically published on February 17, 2009

\title{
MIYAOKA-YAU INEQUALITY FOR MINIMAL PROJECTIVE MANIFOLDS OF GENERAL TYPE
}

\author{
YUGUANG ZHANG
}

(Communicated by Jon G. Wolfson)

\begin{abstract}
In this short paper, we prove the Miyaoka-Yau inequality for minimal projective $n$-manifolds of general type by using Kähler-Ricci flow.
\end{abstract}

\section{INTRODUCTION}

If $M$ is a projective $n$-manifold with ample canonical bundle $\mathcal{K}_{M}$, there exists a Kähler-Einstein metric $\omega$ with negative scalar curvature by Yau's theorem on the Calabi conjecture (14]), which was obtained by Aubin independently (1]). As a consequence, there is an inequality for Chern numbers, the Miyaoka-Yau inequality,

$$
\left(\frac{2(n+1)}{n} c_{2}(M)-c_{1}^{2}(M)\right) \cdot\left(-c_{1}(M)\right)^{n-2} \geq 0,
$$

where $c_{1}(M)$ and $c_{2}(M)$ are the first and the second Chern classes of $M$ (cf. [13]). Furthermore, if the equality in (1.1) holds, the Kähler-Einstein metric $\omega$ is a complex hyperbolic metric; i.e. the holomorphic sectional curvature of $\omega$ is a negative constant. If $n=2$, (1.1) even holds for algebraic surfaces of general type (cf. 4, [8], 9]), which may not admit any Kähler-Einstein metric. In [12, the inequality (1.1) is proved for any dimensional minimal projective manifold of general type by using conic Kähler-Einstein metrics. In this short paper, we give a different proof of (1.1) for minimal projective $n$-manifolds of general type by using Kähler-Ricci flow and study the extremal case of (1.1).

Let $M$ be a minimal projective manifold of general type with $\operatorname{dim}_{\mathbb{C}} M=n \geq 2$. The canonical bundle $\mathcal{K}_{M}$ of $M$ is big, and semi-ample, i.e. $\mathcal{K}_{M}^{n}>0$, and, for a positive integer $m \gg 1$, the linear system $\left|m \mathcal{K}_{M}\right|$ is base point free (as quoted in [11]). For $m \gg 1$, the complete linear system $\left|m \mathcal{K}_{M}\right|$ defines a holomorphic map $\Phi: M \longrightarrow \mathbb{C P}^{N}$, which is birational onto its image $M_{\text {can }} . M_{\text {can }}$ is called the canonical model of $M$, and $\Phi$ is called the contraction map. Note that $M$ may not admit any Kähler-Einstein metric. The Kähler-Ricci flow is an evolution equation of a family of Kähler metrics $\omega_{t}, t \in[0, T)$, on $M$,

$$
\partial_{t} \omega_{t}=-\operatorname{Ric}\left(\omega_{t}\right)-\omega_{t}
$$

Received by the editors October 20, 2008, and, in revised form, November 30, 2008.

2000 Mathematics Subject Classification. Primary 53C55, 53C44.

This work was supported by the SRC program of the Korea Science and Engineering Foundation (KOSEF) grant funded by the Korean government (MEST) (No. R11-2007-035-02002-0), and by the National Natural Science Foundation of China 10771143.

(C)2009 American Mathematical Society 2749

Reverts to public domain 28 years from publication 
where $\operatorname{Ric}\left(\omega_{t}\right)$ is the Ricci form of $\omega_{t}$. By [1], [10, [3], and [15, for any Kähler metric as initial metric, the solution $\omega_{t}$ of the Kähler-Ricci flow equation exists for all time $t \in[0, \infty)$, and the scalar curvature of $\omega_{t}$ is uniformly bounded. Thus we can prove (1.1) by using the technique developed in [6], where a Hitchin-Thorpe type inequality was proved for 4-manifolds which admit a long time solution to a normalized Ricci flow equation with bounded scalar curvature. Before proving the Miyaoka-Yau inequality, we show that the $L^{2}$-norm of the Einstein tensor tends to zero along a subsequence of a solution of the Kähler-Ricci flow equation (1.2).

Theorem 1.1. Let $M$ be a minimal projective manifold of general type with $\operatorname{dim}_{\mathbb{C}} M$ $=n \geq 2$, and let $\omega_{t}, t \in[0, \infty)$, be a solution of the Kähler-Ricci flow equation (1.2). Then there exists a sequence of times $t_{k} \longrightarrow \infty$, when $k \longrightarrow \infty$, such that

$$
\lim _{k \longrightarrow \infty} \int_{M}\left|\rho_{t_{k}}\right|^{2} \omega_{t_{k}}^{n}=0
$$

where $\rho_{t_{k}}=R i c_{t_{k}}-\frac{R_{t_{k}}}{n} \omega_{t_{k}}$ denotes the Einstein tensor of $\omega_{t_{k}}$ and $R_{t_{k}}$ denotes the scalar curvature of $\omega_{t_{k}}$.

As a corollary of this theorem, we obtain the Miyaoka-Yau inequality for minimal projective manifolds of general type.

Corollary 1.2. If $M$ is a minimal projective manifold of general type with $\operatorname{dim}_{\mathbb{C}} M$ $=n \geq 2$, then

$$
\left(\frac{2(n+1)}{n} c_{2}(M)-c_{1}^{2}(M)\right) \cdot\left(-c_{1}(M)\right)^{n-2} \geq 0 .
$$

Furthermore, if the equality holds, there is a complex hyperbolic metric on the smooth part $M_{0}$ of the canonical model $M_{\text {can }}$ of $M$.

\section{Proof of Theorem 1.1}

Let $M$ be a minimal projective manifold of general type with $\operatorname{dim}_{\mathbb{C}} M=n \geq 2$, $M_{\text {can }}$ be the canonical model of $M$, and $\Phi: M \longrightarrow M_{c a n}$ be the contraction map. Consider the Kähler-Ricci flow equation on $M$,

$$
\partial_{t} \omega_{t}=-\operatorname{Ric}\left(\omega_{t}\right)-\omega_{t},
$$

with initial metric $\omega_{0}$. In [7], the short time existence of the solution of (2.1) is proved. Then, in [11, [10], and [3], it is proved that the solution $\omega_{t}$ of (2.1) exists for all time, i.e. $t \in[0,+\infty)$, and there exists a unique semi-positive current $\omega_{\infty}$ on $M$ which satisfies that:

(1) $\omega_{\infty}$ represents $-2 \pi c_{1}(M)$.

(2) $\omega_{\infty}$ is a smooth Kähler-Einstein metric with negative scalar curvature on $\Phi^{-1}\left(M_{0}\right)$, where $M_{0}$ is the smooth part of $M_{\text {can }}$.

(3) On any compact subset $K \subset \Phi^{-1}\left(M_{0}\right), \omega_{t} C^{\infty}$-converges to $\omega_{\infty}$ when $t \longrightarrow \infty$.

In [15, it is shown that there is a constant $C>0$ depending only on $\omega_{0}$ such that

$$
\left|R_{t}\right|<C,
$$

where $R_{t}$ is the scalar curvature of $\omega_{t}$. 
First, we need evolution equations for volume forms and scalar curvatures as follows:

$$
\partial_{t} \omega_{t}^{n}=-\left(R_{t}+n\right) \omega_{t}^{n}
$$

and

$$
\partial_{t} R_{t}=\triangle_{t} R_{t}+\left|R i c_{t}\right|^{2}+R_{t}=\triangle_{t} R_{t}+\left|R i c_{t}{ }^{\circ}\right|^{2}-\left(R_{t}+n\right),
$$

where $R i c_{t}{ }^{\circ}=R i c_{t}+\omega_{t}$ and $\left|R i c_{t}{ }^{\circ}\right|^{2}=\left|R i c_{t}\right|^{2}+2 R_{t}+n$ (cf. Lemma 2.38 in [5] ).

Lemma 2.1. There are two constants $t_{0}>0$ and $c>0$ independent of $t$ such that, for $t>t_{0}$,

$$
\breve{R}_{t}=\inf _{x \in M} R_{t}(x) \leq-n+e^{-t} c<-\frac{n}{2}<0 .
$$

Proof. If we define $\alpha_{t}=\left[\omega_{t}\right] \in H^{1,1}(M, \mathbb{R})$, from (2.1) we have

$$
\partial_{t} \alpha_{t}=-2 \pi c_{1}(M)-\alpha_{t}
$$

and

$$
\alpha_{t}=-2 \pi c_{1}(M)+e^{-t}\left(2 \pi c_{1}(M)+\alpha_{0}\right) \text {. }
$$

Thus

$$
\left[\omega_{\infty}\right]=\alpha_{\infty}=\lim _{t \longrightarrow \infty} \alpha_{t}=-2 \pi c_{1}(M)
$$

Since

$$
\breve{R}_{t} \int_{M} \omega_{t}^{n} \leq \int_{M} R_{t} \omega_{t}^{n}=n \int_{M} R i c_{t} \wedge \omega_{t}^{n-1}=n 2 \pi c_{1}(M) \cdot \alpha_{t}^{n-1},
$$

we obtain

$$
\begin{aligned}
\breve{R}_{t} \leq n \frac{2 \pi c_{1}(M) \cdot \alpha_{t}^{n-1}}{\alpha_{t}^{n}} & =n \frac{2 \pi c_{1}(M) \cdot \alpha_{t}^{n-1}}{-2 \pi c_{1}(M) \cdot \alpha_{t}^{n-1}+e^{-t}\left(2 \pi c_{1}(M)+\alpha_{0}\right) \cdot \alpha_{t}^{n-1}} \\
& =\frac{-n}{1+e^{-t} A_{t}},
\end{aligned}
$$

where $A_{t}=-\frac{\left(2 \pi c_{1}(M)+\alpha_{0}\right) \cdot \alpha_{t}^{n-1}}{2 \pi c_{1}(M) \cdot \alpha_{t}^{n-1}}$. Note that $\left(-c_{1}(M)\right)^{n}>0$. Thus there is a $t_{1}>0$ such that if $t>t_{1}, A_{t}<\left|\frac{\left(\alpha_{\infty}+\alpha_{0}\right) \cdot \alpha_{\infty}^{n-1}}{\alpha_{\infty}^{n}}\right|+1=A$, and we obtain that

$$
\breve{R}_{t} \leq \frac{-n}{1+e^{-t} A}<-n+e^{-t} c
$$

where $c=-n\left(\frac{A}{1+e^{-t_{1} A}}\right)$. By taking $t_{0}>t_{1}$ such that $e^{-t_{0}} c<\frac{n}{2}$, we obtain the conclusion.

\section{Lemma 2.2.}

$$
\int_{0}^{\infty} \int_{M}\left|R_{t}+n\right| \omega_{t}^{n} d t<\infty
$$

Proof. By (2.4) and the maximal principle, $\partial_{t} \breve{R}_{t} \geq-\left(\breve{R}_{t}+n\right)$, and so

$$
n+\breve{R}_{t} \geq C e^{-t}
$$


for a constant $C$ independent of $t$. Note that by Lemma 2.1, (2.7) and (2.5), when $t>t_{0}$

$$
\begin{aligned}
\int_{M}\left|R_{t}+n\right| \omega_{t}^{n} & \leq \int_{M}\left(R_{t}-\breve{R}_{t}\right) \omega_{t}^{n}+\int_{M}\left|n+\breve{R}_{t}\right| \omega_{t}^{n} \\
& \leq \int_{M}\left(R_{t}+n\right) \omega_{t}^{n}+2 \int_{M}\left|n+\breve{R}_{t}\right| \omega_{t}^{n} \\
& \leq \int_{M}\left(R_{t}+n\right) \omega_{t}^{n}+C_{3} e^{-t} \\
& =n\left(2 \pi c_{1} \cdot \alpha_{t}^{n-1}+\alpha_{t}^{n}\right)+C_{3} e^{-t} \\
& =n e^{-t}\left(2 \pi c_{1}+\alpha_{0}\right) \cdot \alpha_{t}^{n-1}+C_{3} e^{-t} \\
& \leq C_{4} e^{-t}
\end{aligned}
$$

for two constants $C_{3}$ and $C_{4}$ independent of $t$. Thus

$$
\int_{0}^{\infty} \int_{M}\left|R_{t}+n\right| \omega_{t}^{n} d t=\int_{0}^{t_{0}} \int_{M}\left|R_{t}+n\right| \omega_{t}^{n} d t+\int_{t_{0}}^{\infty} \int_{M}\left|R_{t}+n\right| \omega_{t}^{n} d t<\infty .
$$

Proof of Theorem 1.1. From (2.4), (2.3), (2.6), (2.2), and Lemma 2.2, we obtain

$$
\begin{aligned}
\int_{0}^{\infty} \int_{M}\left|R i c^{\circ}{ }_{t}\right|^{2} \omega_{t}^{n} d t & =\int_{0}^{\infty} \int_{M}\left(\frac{\partial}{\partial t} R_{t}\right) \omega_{t}^{n} d t+\int_{0}^{\infty} \int_{M}\left(R_{t}+n\right) \omega_{t}^{n} d t \\
& =\int_{0}^{\infty} \frac{\partial}{\partial t}\left(\int_{M} R_{t} \omega_{t}^{n}\right) d t+\int_{0}^{\infty} \int_{M}\left(R_{t}+1\right)\left(R_{t}+n\right) \omega_{t}^{n} d t \\
& \leq n \alpha_{\infty}^{n}-\int_{M} R_{0} \omega_{0}^{n}+C \int_{0}^{\infty} \int_{M}\left|R_{t}+n\right| \omega_{t}^{n} d t \\
& <\infty .
\end{aligned}
$$

If $\rho_{t}=R i c_{t}-\frac{R_{t}}{n} \omega_{t}$ is the Einstein tensor of $\omega_{t}$, then $\left|\rho_{t}\right|^{2}=\left|R i c^{\circ}{ }_{t}\right|^{2}-\frac{1}{n}\left(R_{t}+n\right)^{2}$, and from the above estimation,

$$
\int_{0}^{\infty} \int_{M}\left|\rho_{t}\right|^{2} \omega_{t}^{n} d t \leq \int_{0}^{\infty} \int_{M}\left|R i c^{\circ}{ }_{t}\right|^{2} \omega_{t}^{n} d t<\infty
$$

Thus there is a sequence $t_{k} \longrightarrow \infty$ such that

$$
\lim _{k \longrightarrow \infty} \int_{M}\left|\rho_{t_{k}}\right|^{2} \omega_{t_{k}}^{n}=0
$$

Proof of Corollary 1.2. Note that the Kähler curvature tensor has a decomposition

$$
R m_{t}=\frac{R_{t}}{2 n^{2}} \omega_{t} \otimes \omega_{t}+\frac{1}{n} \omega_{t} \otimes \rho_{t}+\frac{1}{n} \rho_{t} \otimes \omega_{t}+B_{t}
$$

(cf. (2.63) and (2.38) in [2]). By Chern-Weil theory,

$\left(\frac{2(n+1)}{n} c_{2}(M)-c_{1}^{2}(M)\right) \cdot\left[\omega_{t}\right]^{n-2}=\frac{(n-2) !}{4 \pi^{2} n !} \int_{M}\left(\frac{n+1}{n}\left|B_{0, t}\right|^{2}-\frac{\left(n^{2}-2\right)}{n^{2}}\left|\rho_{t}\right|^{2}\right) \omega_{t}^{n}$

(cf. (2.82a) and (2.67) in [2]), where $B_{0, t}=B_{t}-\frac{\operatorname{tr} B_{t}}{n^{2}-1} \operatorname{Id}$ is the tensor given by (2.64) in 2] corresponding to $\omega_{t}$. By Theorem 1.1, there is a sequence $t_{k} \longrightarrow \infty$ such that

$$
\lim _{k \longrightarrow \infty} \int_{M}\left|\rho_{t_{k}}\right|^{2} \omega_{t_{k}}^{n}=0 .
$$


Hence

$$
\begin{aligned}
\left(\frac{2(n+1)}{n}\right. & \left.c_{2}(M)-c_{1}^{2}(M)\right) \cdot\left(-2 \pi c_{1}(M)\right)^{n-2} \\
& =\left(\frac{2(n+1)}{n} c_{2}(M)-c_{1}^{2}(M)\right) \cdot\left[\omega_{\infty}\right]^{n-2} \\
& =\lim _{k \longrightarrow \infty}\left(\frac{2(n+1)}{n} c_{2}(M)-c_{1}^{2}(M)\right) \cdot\left[\omega_{t_{k}}\right]^{n-2} \\
& =\lim _{k \longrightarrow \infty} \frac{(n-2) !}{4 \pi^{2} n !} \int_{M}\left(\frac{n+1}{n}\left|B_{0, t_{k}}\right|^{2}\right) \omega_{t_{k}}^{n} \\
& \geq 0 .
\end{aligned}
$$

If the equality holds, on any compact subset $K \subset \Phi^{-1}\left(M_{0}\right)$,

$$
\int_{K}\left|B_{0, \infty}\right|^{2} \omega_{\infty}^{n} \leq \lim _{k \longrightarrow \infty} \int_{M}\left|B_{0, t_{k}}\right|^{2} \omega_{t_{k}}^{n}=0
$$

by the smooth convergence of $\omega_{t}$ to $\omega_{\infty}$. Thus $B_{0, \infty} \equiv 0$. Since $\omega_{\infty}$ is a KählerEinstein metric with negative scalar curvature on $\Phi^{-1}\left(M_{0}\right)$, the holomorphic sectional curvature is a negative constant by Section 2.66 in [2]; i.e. $\omega_{\infty}$ is a complex hyperbolic metric.

\section{ACKNOWLEDGEMENTS}

The author thanks Zhou Zhang for explaining [15] and for sending him the paper [10. Thanks also go to the referees for their suggestions on improving the present paper. The author also thanks Valentino Tosatti for sending him [12].

\section{REFERENCES}

1. T. Aubin, Équations du type Monge-Ampère sur les variétés kähleriennes compactes. C. R. Math. Acad. Sci. Paris Sér. A-B, 283 (1976), A119-A121. MR0433520 (55:6496)

2. A. L. Besse, Einstein manifolds. Ergebnisse der Math., Springer-Verlag, Berlin-New York, 1987. MR867684 (88f:53087)

3. P. Cascini, P. La Nave, Kähler-Ricci flow and the minimal model program for projective varieties. arXiv:math/0603064.

4. S.Y. Cheng, S.-T. Yau, Inequality between Chern numbers of singular Kähler surfaces and characterization of orbit space of discrete group of $\mathrm{SU}(2,1)$. Contemporary Math., 49, Amer. Math. Soc., Providence, RI, 1986, 31-44. MR833802 (87m:53078)

5. B. Chow, S.-C. Chu, D. Glickenstein, C. Guenther, J. Isenberg, T. Ivey, D. Knopf, P. Lu, F. Luo, L. Ni, The Ricci Flow: Techniques and Applications, Part I: Geometric Aspects. Mathematical Surveys and Monographs, Vol. 135, Amer. Math. Soc., Providence, RI, 2007. MR.2302600 (2008f:53088)

6. F. Fang, Y. Zhang, Z. Zhang, Non-singular solutions to the normalized Ricci flow equation. Math. Ann., 340 (2008), 647-674. MR2357999 (2009a:53112)

7. R. Hamilton, Three-manifolds with positive Ricci curvature. J. Diff. Geom., 17 (1982), 255306. MR664497 (84a:53050)

8. R. Kobayashi, Einstein-Kähler V-metrics on open Satake V-surfaces with isolated quotient singularities. Math. Ann., 272, no. 3 (1985), 385-398. MR799669 (87b:32050)

9. Y. Miyaoka, On the Chern numbers of surfaces of general type. Invent. Math., 42 (1977), 225-237. MR0460343(57:337)

10. G. Tian, Z. Zhang, On the Kähler-Ricci flow on projective manifolds of general type. Chinese Annals of Mathematics Ser. B, 27 (2006), 179-192. MR2243679 (2007c:32029)

11. H. Tsuji, Existence and degeneration of Kähler-Einstein metrics on minimal algebraic varieties of general type. Math. Ann., 281 (1988), 123-133. MR944606 (89e:53075)

12. H. Tsuji, Stability of tangent bundles of minimal algebraic varieties, Topology, 27, no. 4 (1988), 429-442. MR0976585 (90d:14021) 
13. S.T. Yau, Calabi's conjecture and some new results in algebraic geometry. Proc. Natl. Acad. Sci. USA, 74 (1977), 1798-1799. MR0451180 (56:9467)

14. S.T. Yau, On the Ricci curvature of a compact Kähler manifold and the complex MongeAmpère equation. I. Comm. Pure Appl. Math., 31 (1978), 339-411. MR480350(81d:53045)

15. Z. Zhang, Scalar Curvature Bound for Kähler-Ricci Flows over Minimal Manifolds of General Type. arXiv:math/0801.32481.

Department of Mathematics, Capital Normal University, Beijing, People's Republic OF CHINA

Current address: Department of Mathematical Sciences, Korea Advanced Institute of Science and Technology, Daejeon, Republic of Korea

E-mail address: yuguangzhang76@yahoo.com 